\title{
Numerical modelling of combined roughness and plasticity induced crack closure effects in fatigue
}

\author{
M.R. Parry ${ }^{a, *}$, S. Syngellakis ${ }^{b}$, I. Sinclair ${ }^{a}$ \\ ${ }^{a}$ Materials Research Group, School of Engineering Sciences, University of Southampton, Southampton, SO17 1BJ, UK \\ ${ }^{\mathrm{b}}$ Computational Mechanics and Design Group, School of Engineering Sciences, University of Southampton, Southampton, SO17 1BJ, UK
}

Received 23 December 1999; received in revised form 26 March 2000

\begin{abstract}
The incidence of roughness induced fatigue crack closure has been studied by finite element modelling. Closure analyses in the literature have been reviewed and been shown to lack a reasonable treatment of: (a) propagating elastic-plastic cracks, and (b) the influence of the characteristically inhomogeneous plastic deformation associated with near-threshold crack growth in many materials. Based on an analysis of both overall specimen compliance and node behaviour along the crack path, the present modelling shows: (a) an increasing effect of crack path angle on roughness induced closure levels in keeping with the simple analytical model of Suresh and Ritchie; (b) the mechanism by which closure occurs is more strongly dependent on residual plastic strains in the wake than global shear displacements of the fracture surfaces due to mixed-mode behaviour at the crack tip; and (c) the closure levels are relatively low compared to experimental data, consistent with the absence of environmental irreversibility in the finite element models and the idealised crack path morphologies that were studied. Slip band simulations show a significant increasing effect of inhomogeneous deformation on closure levels, improving the apparent accuracy of the modelling results. (C) 2000 Elsevier Science S.A. All rights reserved.
\end{abstract}

Keywords: Fatigue; Elastic-plastic finite element analysis; Roughness induced crack closure; Slip bands

\section{Introduction}

The phenomenon of fatigue crack closure is widely considered to have a strong influence on fatigue crack growth. As such, directed alloy development, accurate life prediction and the implementation of economic inspection procedures in engineering structures are dependent upon the ability to quantify this effect $[1,2]$. Many aluminium aerospace alloys exhibit microscopically deflected crack growth modes, with various aspects of fatigue crack growth then being rationalised by the associated incidence of roughness induced crack closure (RICC) [3]. Whilst numerous experimental studies and modelling exercises on crack closure have been reported over the last 30 years, significant controversy remains. For example, Vasudevan and co-workers [4,5] have suggested that the actual influences of closure on

\footnotetext{
* Corresponding author. Tel.: + 44-2380-595101; fax: +44-2380 593016.

E-mail address: mrp2@soton.ac.uk (M.R. Parry).
}

crack growth are dramatically lower than many articles in the literature suggest, and propose alternative explanations to observed crack growth phenomena. An extensive body of experimental evidence and theoretical analysis does however exist to support the dependence of fatigue crack growth on closure phenomena. Several quantitative and semiquantitative models of RICC exist within the literature, although they are generally rather simplified. Furthermore, interactions between different closure mechanisms are largely ignored. The present work seeks to extend current quantitative understanding, focusing on geometrical and micromechanical closure effects, for a propagating crack in an elasto-plastic material.

\subsection{Background}

The first discovery of an extrinsic contribution to fatigue crack growth resistance (i.e. due to mechanical shielding of the crack tip, as opposed to intrinsic microstructural resistance to crack growth) is usually at- 

tributed to Elber [6]. Elber noted that as a fatigue crack propagates, plastically stretched material in the crack wake leads to premature contact of the crack faces, a process referred to as plasticity induced crack closure (PICC). Other mechanisms by which shielding of the crack tip can occur have since been identified (e.g. see Ref. [7]). The possibility that crack path roughness may lead to premature crack closure was first identified by Walker and Beevers [8], who studied fatigue crack growth in titanium samples and found that whilst PICC effects were apparently absent, contact occurred at discrete points behind the crack tip as a result of deflected crack growth and the associated shear of the crack flanks. In order for RICC to occur a combination of crack path deflection and a residual shear offset of the fracture surfaces is clearly required. Crack deflection can occur along grain boundaries, slip bands, or at secondary phases. At the tip of a deflected crack mixed mode loading conditions will exist, which may then lead to a global shear offset of the fracture surfaces due to irreversibility of the local plastic deformation. In planar slip materials plastic deformation can be highly localised to bands of intense shear ahead of the crack tip [9]. Crack growth may then occur by shear decohesion along the slip bands. Thus the crack path will follow well defined crystallographic directions, and lead to highly faceted fracture surfaces. The incidence of slip band crack growth is illustrated in Fig. 1 for a Ni based superalloy. The very marked shear strain concentration of the slip bands may be seen in the offsets in the etched $\gamma^{\prime}\left(\mathrm{Ni}_{3} \mathrm{Al}\right)$ precipitates along the individual slip bands. Whilst PICC is generally considered to be most pronounced under plane stress conditions, high levels of crack closure have been reported for plane strain, near threshold conditions [10,11]. Such results have been related to high levels of RICC due to extensive slip band crack growth in the near threshold regime.

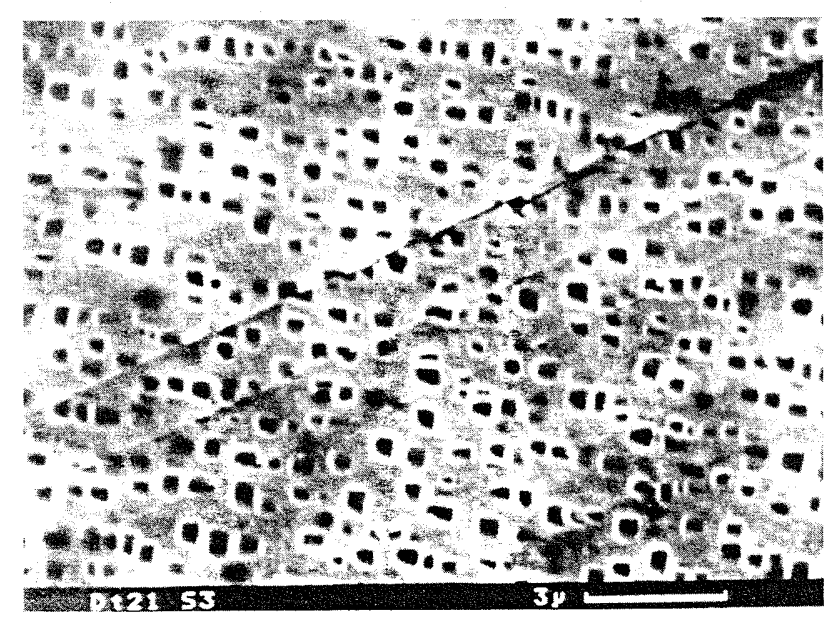

Fig. 1. Slip band crack growth in a Ni-alloy showing marked shear strain localization in bands ahead of the propagating crack tip.

\subsection{Finite element modelling of PICC}

Initial attempts to model PICC using the finite element method were presented independently by Newman and Armen [12] and Ohji et al. [13]. Using an essentially arbitrary crack growth algorithm and continuum plasticity theory, a finite element model of a cracked plate was constructed. It was predicted that the crack faces come into contact under tensile far field loads in agreement with experimental observations. Although these analyses were limited to a small number of crack growth increments, steady-state closure levels were predicted under plane stress conditions in reasonable agreement with experimental results. In particular, the effects of variable amplitude loading histories and $R$-ratio were investigated. Similar models have subsequently been used by many researchers to investigate closure under different stress states and loading conditions (e.g. Refs. [14-28]). It is apparent from the various numerical models which have been presented in the literature that there are several important issues, such as mesh sufficiency and the attainment of a steady-state crack closure level, which must be addressed to produce meaningful finite element models of the crack closure process. A detailed review of these issues is presented by McClung and Sehitoglu [14].

\subsection{Modelling PICC under plane strain}

The issue of PICC under plane strain conditions has been the subject of some debate (e.g. see Refs. [29-31]). Under plane stress, through-thickness contraction is a clear source of the 'extra material' required for crack closure to occur. Under plane strain conditions through-thickness contraction is zero by definition, and this has led various authors to argue that PICC cannot occur under these conditions [5,32]. However, there are experimental data to suggest that PICC can occur under plane strain conditions [33,34]. This was first investigated through the use of numerical models by Ogura et al. [15] and Blom and Holm [16]. The results of Blom and Holm in particular showed stabilised closure levels in agreement with experimental results

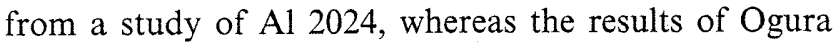
et al. would not satisfy the mesh refinement criteria of McClung and Sehitoglu [14], and so may be regarded as questionable. Fleck and Newman [17] argued that PICC in plane strain was due to an artificial 'wedge' of material forming near the pre-crack tip used in the modelling process. It was argued that if the crack was allowed to grow far away from this wedge, then its influence would diminish such that closure levels would go to zero, i.e. plane strain PICC essentially being a transient effect caused by unrepresentative residual strains at the pre-crack. This, however, is in apparent contradiction with more recent studies [18-21], which 


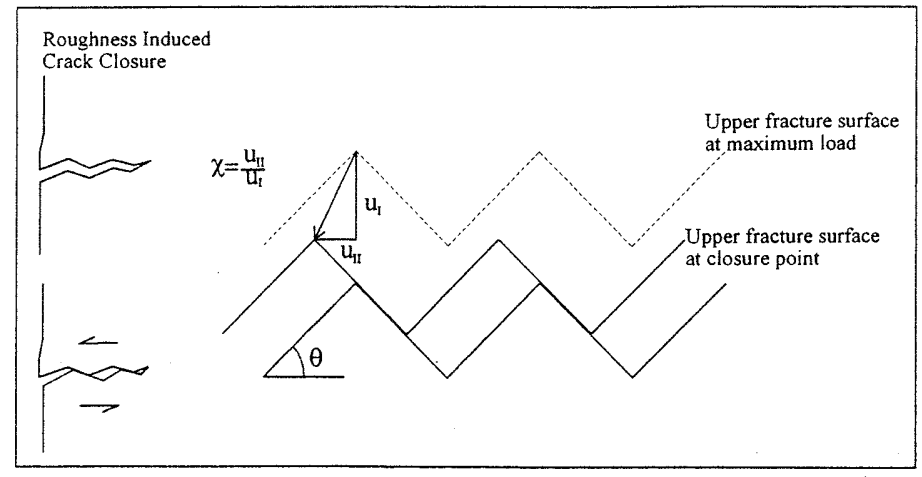

Fig. 2. Detail of the geometrical model of roughness induced crack closure of Suresh and Ritchie [30].

have shown plane strain closure levels in the region of 0.2 to $0.3 K_{\max }$, for $R=0$, under small-scale yielding conditions. The origin of this closure has been shown to be a contraction of material in the in-plane transverse direction, (i.e. parallel to the crack plane). This is consistent with the analytical descriptions of plane strain closure subsequently put forward by Pippan and Riemelmoser [29,30].

However, fundamental to the closure levels reported in the literature are the ways in which the closure point is defined. Newman and Armen's [12] initial approach was to monitor the behaviour of the first node behind the tip, and to define the closure point as the point in the load cycle at which the displacement of the node immediately behind the crack tip from the crack symmetry plane becomes zero. This approach has been adopted in many subsequent analyses in the literature. However, Fleck and Newman [17] showed that the node immediately behind the crack tip may be affected by unrealistic residual strains close to the tip (arising from the crack extension process). In particular, the contact behaviour of the first node behind the tip can be very different from the rest of the nodes behind the crack tip, regardless of the mesh size, (i.e. exhibiting clear mesh dependency). Hence, it was suggested that closure at the first node behind the tip should not be taken as the definition of the closure point. Attempts to identify crack closure points based on nodal contact are, of course, subject to the essentially nominal scale of mesh refinement used, and attempts have been made $[22,23]$ to avoid such definitions by examining instead changes in specimen compliance (cf. experimental closure determination methods). However, no comparisons between the closure points determined by the compliance method and by the behaviour of nodes behind the crack tip were apparently made. Overall there does not appear to be any standard definition of the closure point in finite element analyses. For example, although McClung and Sehitoglu [14] adopted the criterion of the closure at the second node behind the crack tip, the original criterion of closure at the first node behind the crack tip is used in a later paper by Sehitoglu and Sun [19].

\subsection{RICC specific models}

Numerous attempts have been made to model RICC effects (e.g. Refs. [35-38]). Suresh and Ritchie [35] derived an expression for the closure stress intensity factor for RICC using a simple geometrical model of a deflecting crack:

$\frac{K_{\mathrm{cl}}}{K_{\max }}=\sqrt{\frac{\chi \tan \theta}{1+\chi \tan \theta}}$

where $\theta$ is the crack deflection angle and $\chi$ is the ratio of the mode II to the mode I displacement that occurs during unloading, illustrated in Fig. 2. In keeping with the basic link between surface geometry and closure levels Wasen et al. [36] proposed an empirical relationship between $K_{\mathrm{cl}}$ and the standard deviation of asperity heights, based on experimental observations of ferritic steels.

Wang et al. [37] have argued that the Suresh model is geometrically oversimplified, and that the empirical relationship of Wasen et al. contains no information on the applied load or the asperity shape. Hence, they have extended these statistical and geometric approaches by incorporating a dislocation-based model to estimate fracture surface mismatch. However the predictive power of this model is limited due to the inclusion of unknown proportionality constants, which must be fitted to experimental results. Similarly, the $\chi$ parameter used in the Suresh and Ritchie model is essentially a fitting parameter.

Llorca [38] used the finite difference technique to demonstrate the effect of periodic and irregular crack deflection for low $\Delta K$ plane strain conditions. It was shown that increasing levels of crack closure occur with increasing crack deflection angle, which are further enhanced by the varying deflection angle along a given crack path. As noted earlier, for crack roughness to enhance closure levels, some residual shear offset of the 
fracture surfaces must exist. The only source of this offset can be plasticity effects at the crack tip. However, these do not appear to be fully addressed in Llorca's work. In particular, there is no refinement of the mesh to accurately account for the expected scale of crack tip plasticity.

\subsection{Scope and objectives of the present work}

The present work is concerned with the finite element modelling of crack closure, arising from the combined effects of crack deflection and prior plastic deformation, in a long fatigue crack in an aerospace aluminium alloy under constant amplitude cyclic loading such that small scale yielding conditions exist at the crack tip. This has been done through an extension of the fatigue crack growth modelling concepts of Newman and Armen [12] to the case of a periodically deflected crack. Continuum plasticity theory has been used with attention being paid to accurate modelling of crack tip plasticity in line with McClung and Sehitoglu's recommendations [14]. Issues in the definition of the closure point from the literature have been investigated through combined monitoring of crack face contact and specimen compliance in models of undeflected cracks. The underlying mechanism leading to the observed RICC has been identified through the isolation of the effects of deflected crack propagation and the effects of plastic deformation at the crack tip. Additionally, a novel approach has been taken to simulate the localisation of strain within the planar slip bands ahead of the crack tip, and the associated influences on shear offsets during crack growth.

\section{Development of the finite element models}

\subsection{Basic continuum models}

The general purpose finite element code ABAQUS [39] was used to model a standard centre cracked plate (CCP) specimen geometry, as defined by ASTM E647 [40], for width $W=75 \mathrm{~mm}$, initial crack length $a_{0}=7.6$ $\mathrm{mm}$, thickness $B=7.5 \mathrm{~mm}$, and notch height $h=1 \mathrm{~mm}$. Symmetry considerations allowed one half of the specimen to be modelled for deflected crack growth (one quarter for the undeflected crack models). Material properties analogous to a damage tolerant aluminium alloy were chosen, i.e. yield stress $\sigma_{0}=370 \mathrm{MPa}$, Young's modulus $E=74 \mathrm{GPa}$, hardening modulus $H=0.07 E$, Poisson's ratio $v=0.33$. These were implemented in a standard linear kinematic hardening model. Approximately 10234 first order isoparametric quadrilateral elements were used to discretise one half of the CCP specimen (5000 elements in the quarter model). These elements utilise the selectively reduced-integra- tion technique, which helps to prevent mesh locking and provides an accurate solution in incompressible or nearly incompressible cases, see Refs. [39,41]. The mesh was designed such that the element dimension, $L_{\mathrm{e}}$, along the crack line was $1 \mu \mathrm{m}$, with the crack allowed to grow up to $100 \mu \mathrm{m}$. McClung and Sehitoglus' criterion [14] for mesh sufficiency requires that $L_{\mathrm{e}} \leq 0.1 r_{\mathrm{p}}$, where $r_{\mathrm{p}}$ is the forward plastic zone size at maximum load. This requirement means that the adopted $L_{\mathrm{e}}$ is acceptable for a minimum $r_{\mathrm{p}}$ of $10 \mu \mathrm{m}$. In order to obtain stabilised crack closure, experimental results [42] suggest that the crack should be allowed to grow through the equivalent of four plastic zone sizes, giving a maximum $r_{\mathrm{p}}$ of $25 \mu \mathrm{m}$. Under plane strain conditions, this range of $r_{\mathrm{p}}$ (from 10 to $25 \mu \mathrm{m}$ ) equates to $\Delta K=$ 3.6-5.7 $\mathrm{MPa} \mathrm{m} \mathrm{m}^{1 / 2}$ which corresponds to the nearthreshold fatigue regime in which RICC processes are prominent. Four different crack geometries were modelled; an undeflected crack, and cracks undergoing periodic deflections of 30,45 and $60^{\circ}$.

A procedure for incremental crack propagation was developed along the lines of Newman's work $[12,17]$. Pairs of opposite nodes along the crack line were initially connected by two (very short) linear spring elements. The first spring element had no stiffness in compression but was very stiff in tension. The second spring element was very stiff in compression, but had no stiffness in tension, with the spring stiffness acting normal to the crack face so as to prevent crack face interpenetration without affecting the relative shear displacement of the crack faces. Crack propagation was simulated by removing the tension spring element at the crack tip node at maximum load. This allowed the crack to grow one element dimension as the original crack tip nodes were no longer constrained in tension. To assess the role of plastic deformation on the RICC, process modelling was also carried out on a non-propagating deflected crack, and a deflected crack with loading and unloading applied at each crack turning point. Friction effects during contact of the crack surfaces were not considered here.

\subsection{Slip band simulation}

To simulate the presence of slip heterogeneity associated with planar slip bands ahead of a crack tip, the properties of the tension spring elements ahead of the propagating crack tip, were modified to restrain the nodes normal to the line of the crack only, i.e. shear displacement of the nodes could then occur. To restrain the nodes in shear, additional rigid-perfectly plastic truss elements were inserted. By controlling the yield stress of these truss elements, the effective critical resolved shear stress (CRSS) along the crack propagation direction was varied. The CRSS of the slip band plane of area $A_{\text {band }}$ was represented by $n$ truss elements of 
cross sectional area $A_{\text {truss }}$. Hence an area of $A_{\text {band }} / n$ was associated with each truss element. $A_{\text {truss }}$ was set to be equal to $A_{\text {band }} / n$, thus the yield stress of the truss element was equal to the CRSS of the plane. To simulate a local strain softening of the material in the slip band, the yield stress of the truss element was set to

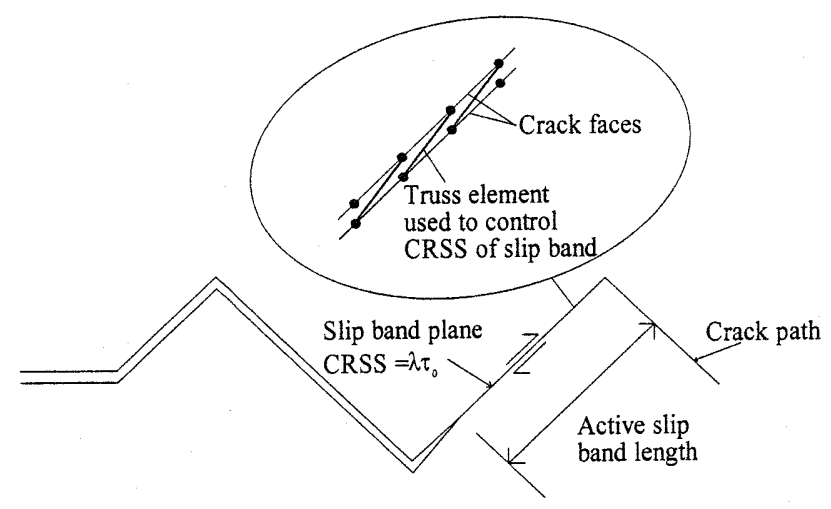

Fig. 3. Schematic diagram showing the simulation of a slip band ahead of a crack.
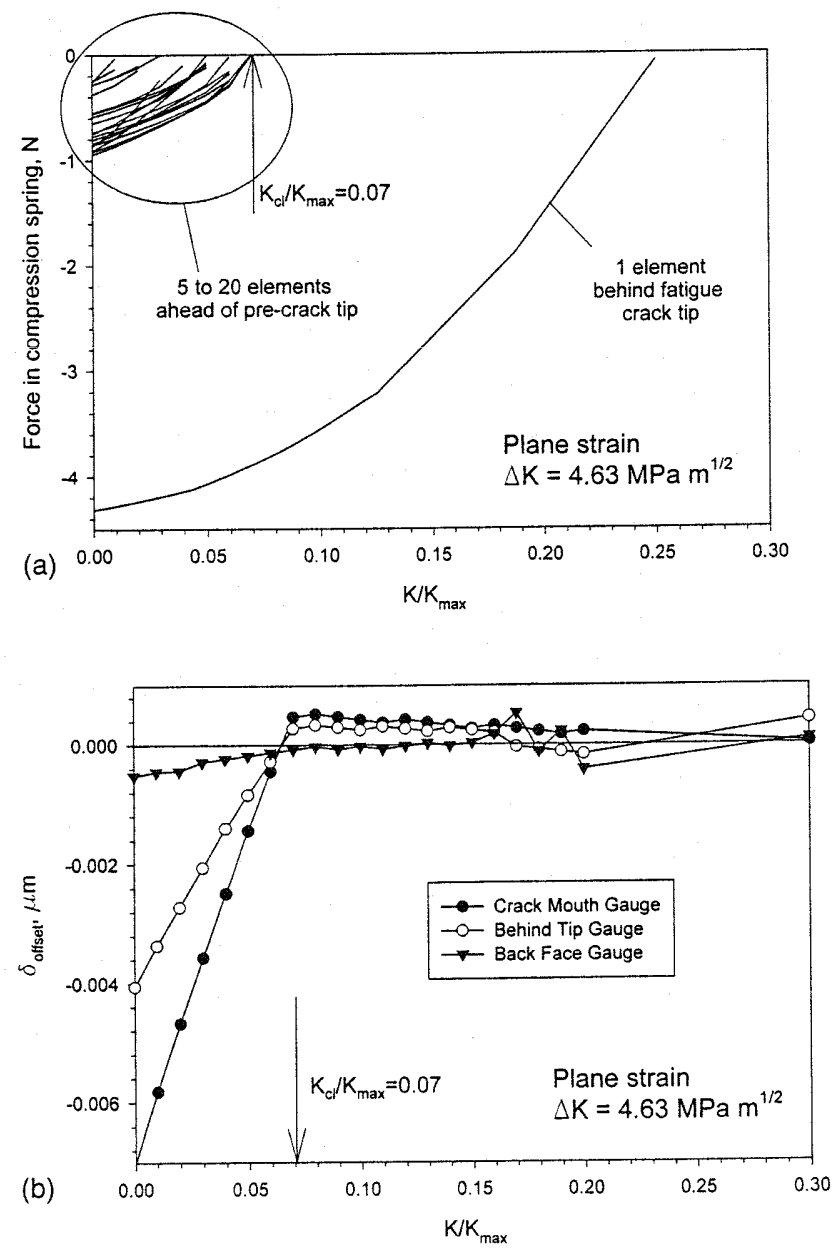

Fig. 4. (a) Response of the spring elements behind the crack tip for an undeflected crack in plane strain. (b) Offset compliance plot for an undeflected crack in plane strain. $\lambda \tau_{0}$, where $\lambda$ is a constant specifying the relative strength of the slip band and bulk material (i.e. $\lambda<1$ for slip band softening) and $\tau_{0}$ is the bulk CRSS $\left(=\sigma_{0} / 2\right)$. The construction of the slip band model is illustrated in Fig. 3. It should be noted that in this model the geometry of the slip band is defined by the geometry of the predefined crack path (i.e. secondary slip bands are not included), and the single band that extends ahead of the crack tip at any point is constrained to the distance to the next (pre-determined) deflection point.

\subsection{Closure assessment}

Closure of the crack was determined by monitoring the forces in the compression spring elements behind the tip, in conjunction with the specimen compliance (i.e. plotting normalised stress intensity factor, $K / K_{\max }$, versus displacement, $\delta$ ) at various locations analogous to the clip/strain gauges used in experimental closure determination, i.e. at the crack mouth, just behind the crack tip, and on the back face of the specimen. The offset compliance method was used to aid closure determination, i.e. a line fit was made to the upper linear part of the compliance curve which was then subtracted from the observed compliance for the entire load cycle. Mathematically, if $m_{\text {open }}$ is the gradient of that section of the compliance curve for which the crack is open, then $\delta_{\text {offset }}=\delta-m_{\text {open }} K / K_{\max }$. The sensitivity of this method depends on the number of decrements in the unloading cycle. Typically, the specimen was unloaded by decrements of one tenth of the applied load, until closure was considered imminent (based on preliminary model runs). Further unloading was then performed using decrements of $0.025-0.01$ of the applied load.

\subsection{Verification of the model}

Extensive verification of the finite element model was performed. In particular, the response of the model to variations in spring stiffness, hardening model (bilinear isotropic, kinematic, and elastic-perfectly plastic), mesh density, and the timing of the nodal release (crack growth), were all investigated. In the interests of brevity, these results are not produced here, but are similar to findings published in the literature [14]. The ability of the chosen elements to correctly model incompressible plane strain deformation was also verified.

\section{Results and discussion}

In Fig. 4 the response of the spring elements behind the crack tip and the associated offset compliance plots are shown for an undeflected crack grown under constant applied stress by $100 \mu \mathrm{m}$, equivalent to six plastic 


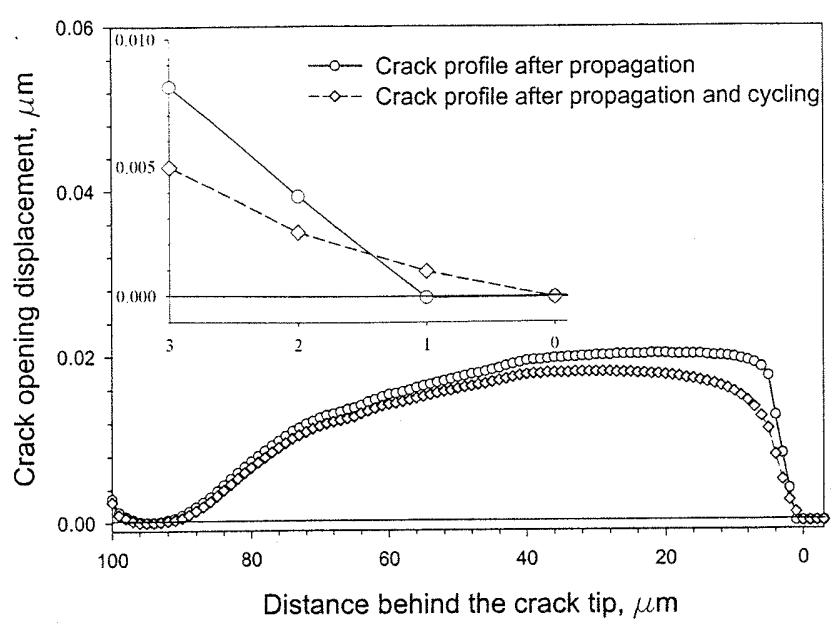

Fig. 5. Crack profile at $K=K_{\mathrm{cl}}=0.07 K_{\max }$, for a propagated crack, and for a propagated crack with two load/unload cycles applied on reaching the final crack length. Inset shows near tip behaviour.

zone diameters, for plane strain conditions. From Fig. $4 \mathrm{a}$ it can be seen that the first spring element behind the crack tip goes into compression (i.e. the crack tip is closed at that point) at 0.25 of the maximum stress intensity factor, $K_{\max }$. Compression of spring elements further behind the crack tip does not occur until significantly later in the unloading process $\left(K / K_{\max }=0.07\right)$, when the crack becomes closed a short distance (5-20 $\mu \mathrm{m})$ ahead of the pre-crack tip, whilst remaining open closer to the tip (see Fig. 5). By considering Fig. $4 \mathrm{~b}$ it is evident that closure at the first node behind the crack tip does not affect the overall specimen compliance. However a deviation in this plot occurs at $K / K_{\max }=$ 0.07 , coinciding with the contact further behind the crack tip. Furthermore, the particular behaviour of the first node behind the tip can be eliminated by repeatedly cycling the crack between maximum and minimum load without any crack propagation. After two such cycles the first node behind the tip no longer comes into contact at all. However, the remaining contact in the crack wake is relatively unaffected by this procedure, as shown in Fig. 5. As such the current models support the view of Fleck and Newman [17] that closure processes associated with the first node behind the tip in a finite element model of a propagating crack may in fact be an artefact of the crack propagation process required in such models.

Assessing the significance of contact at the first node behind the crack tip in relation to the results in the literature is difficult as many authors fail to provide information on the manner of crack face contact in their models [16,20,24-26]. For those authors who have published this information the results of Fleck and Newman [17], Fleck [27], Biner et al. [28] and Wei and James [21] all indicate that anomalous contact of the first node behind the tip occurs in plane strain models of PICC. Progressive plane strain PICC behaviour (i.e. with nodal contact building up along several nodes behind the crack tip during unloading) has been reported by Sehitoglu and Sun [19] and McClung et al. [18] when using a specific finite element code and model formulation. To the authors knowledge there are no other references which report such 'zipping up' behaviour of PICC under plane strain conditions. McClung et al. [18] note that anomalous single node contact behind the crack tip may be attributable to element 'locking' problems in modelling the stress gradients at a plane strain crack tip. This cannot be the case, however, as element locking was not detected in the present analyses (attributable to the use of selectively reduced-integration elements). The present work would, therefore, support the assertion of Fleck [17,27] that very near tip closure effects in plane strain finite element models of PICC may be anomalous and should be treated with caution.

The effect of periodic crack deflection on $K_{\mathrm{cl}} / K_{\max }$ under plane strain loading at $\Delta K=4.63 \mathrm{MPa} \mathrm{m}^{1 / 2} \mathrm{can}$

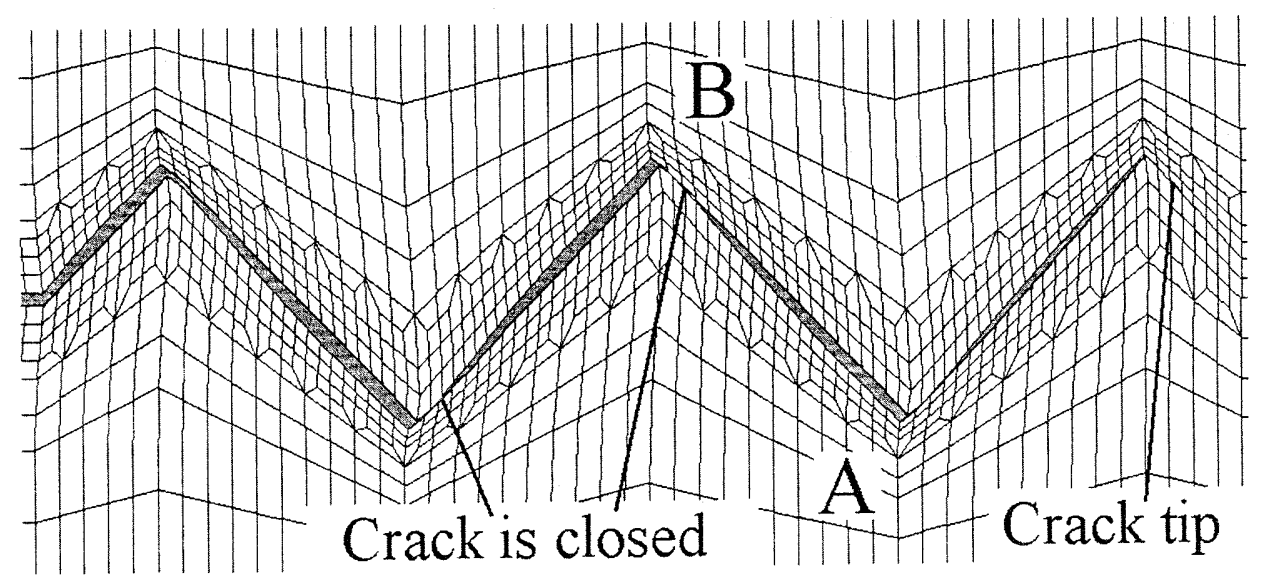

Fig. 6. Plot of the deformed mesh of a $45^{\circ}$ deflected crack at $K=K_{\mathrm{cl}}$, exhibiting closure near the tips of the asperities. Displacement magnification $\times 5$. 

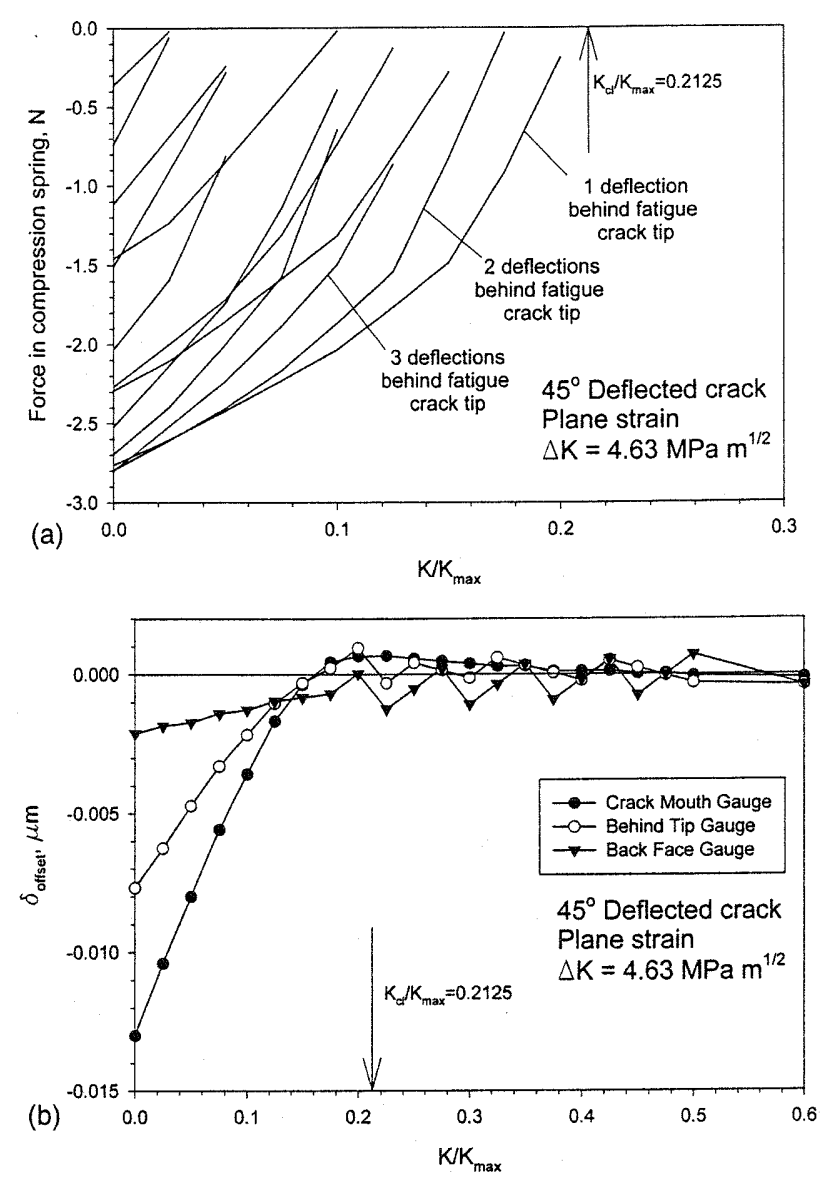

Fig. 7. (a) Response of the compression spring elements behind the crack tip for a $45^{\circ}$ deflected crack. (b) Offset compliance plot for a $45^{\circ}$ deflected crack.

be seen from the plot of the deformed mesh of a $45^{\circ}$ deflected crack at $K<K_{\mathrm{cl}}$ in Fig. 6. Closure can be seen to have occurred at discrete points near the asperity tips, with the bulk of the crack remaining open, as noted by Walker and Beevers [8]. The response of the spring elements behind the tip and the offset compliance plot is shown in Fig. 7. The first spring elements that go into compression (i.e. the points of first contact) are those at the tips of previous crack deflections (i.e. at the asperities), which coincides with the onset of nonlinearity in the offset compliance. As such it would appear that the discontinuous contact problem and the above discussion on crack closure point definition in undeflected plane strain cracks does not influence the behaviour of the deflected cracks and it is, therefore, assumed that the deflected crack closure behaviour illustrated in Fig. 6. is indeed physically realistic. Given the clear build up of crack contact with nonlinearity in compliance, the closure point in these models will be defined in terms of first asperity contact.

From Fig. 8 it is evident that the closure levels increase strongly with deflection angle. It appears that quasi-stabilised levels of closure are reached when the crack has propagated through the first two deflections. The closure levels are at a maximum immediately following a deflection, and then drop off steadily as the crack tip moves away from the point of deflection.

An important observation from these results is the sense of the shear displacements giving rise to asperity contact. In particular it may be seen that the direction of the relative displacements of the upper and lower fracture surfaces at each asperity tip changes along the crack wake. At point ' $A$ ' in Fig. 6 the lower fracture surface is displaced away from the crack tip (in relation to the upper fracture surface), whilst at point ' $B$ ' it is displaced towards the crack tip. It may then be seen that the shear displacements giving rise to closure along the crack wake cannot be 'global' displacements of the upper and lower fracture surfaces due to mixed mode behaviour at the active crack tip. The asperity shear displacements in Fig. 6 can in fact only arise from local residual strains from the crack propagation process. The asperity displacements and contacts observed in the present models are, therefore, somewhat different to the conventional representation of RICC (as illustrated by the Suresh model in Fig. 2). The behaviour of the present models may be rationalised as follows: when a simple deflected crack tip is loaded as shown in Fig. 9a, a permanent plastic shear deformation is produced in the direction/sense shown. On unloading, a degree of reverse plasticity will occur, although a net residual deformation will remain in the direction of the original loading (Fig. 9b). As such, the crack tip will held in a 'compressive' shear (of opposite sense to the loading shear) by the surrounding elastic material when unloading occurs, exactly analogous to the compressive load generated by crack tip plasticity when a simple mode I crack is unloaded. When the crack undergoes a deflection, as shown in Fig. 9c, this residual plastic strain/ compressive loading will always promote closure on the forward edge of each asperity tip, as seen in Fig. 6 .

To illustrate the above process, various simplified models were investigated, see Figs. 10 and 11. Fig. 10 illustrates the unloaded condition of a deflected crack where the crack path was simply 'cut' without a propagation process, with the crack then being loaded and unloaded once, with the resultant plastic deformation producing a degree of shear offset along the asperities. The shear is of identical direction for all asperities, consistent with deformation at the tip being the only source of shear offset, but is in fact insufficient for closure to occur due to the associated residual opening displacements (displacements are greatly magnified in the diagram). In Fig. 11 the crack has been propagated with loading and unloading only being applied at each crack turning point. For the final crack length shown the crack is simply loaded and unloaded elastically, i.e. no shear offset can be generated at the tip. Closure behaviour is seen to be closely analogous to that in Fig. 


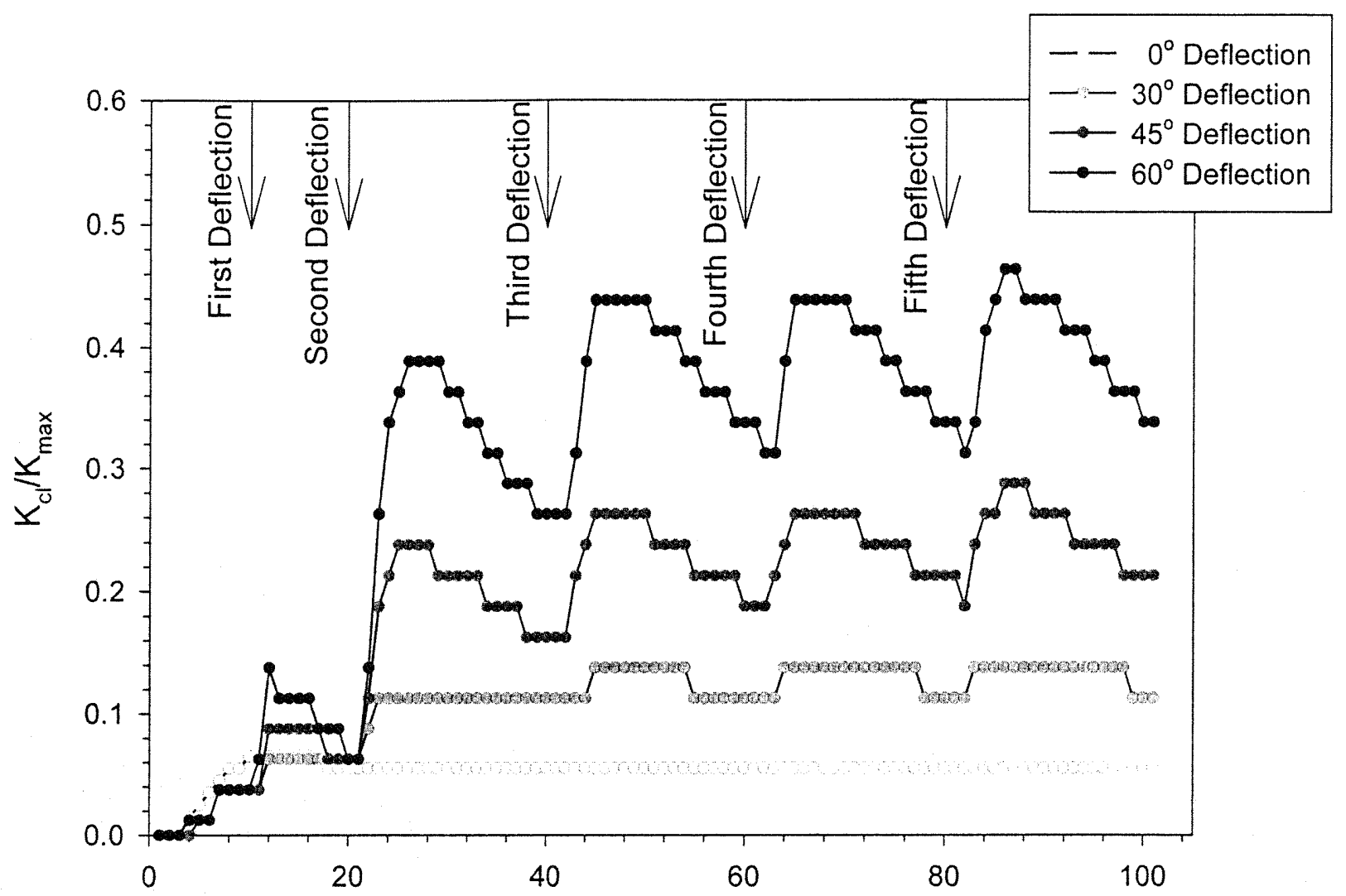

Fig. 8. Variation of the normalised closure stress intensity factor with crack length for different crack geometries.

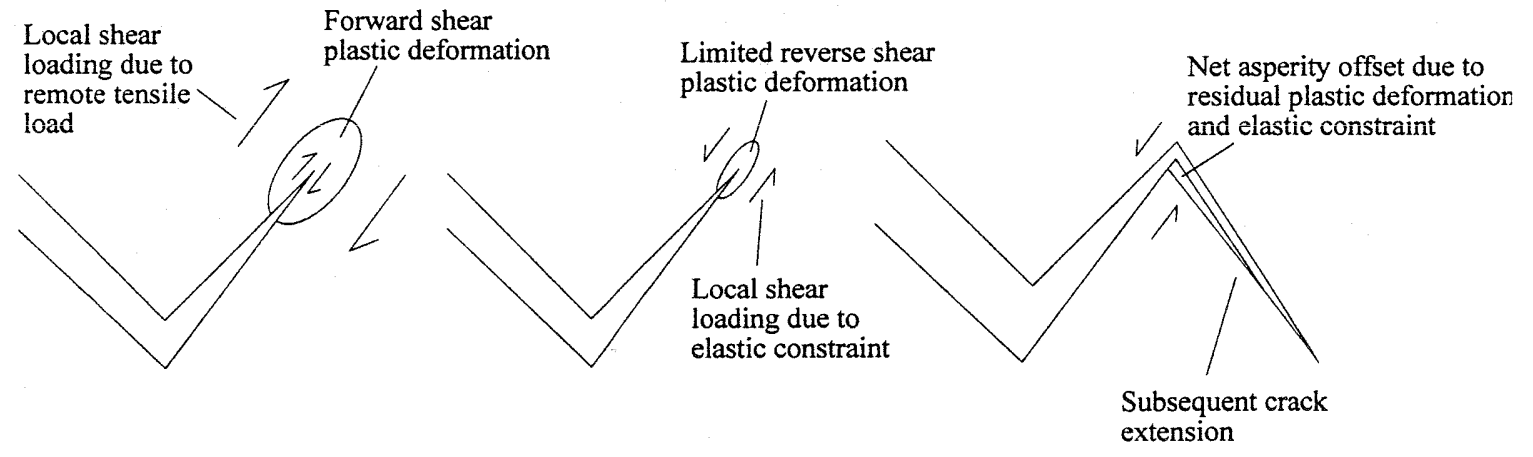

(a) Loaded

(b) Unloaded

(c) Propagation

Fig. 9. Mechanism of crack closure due to residual shear deformation in the crack wake.

6 , confirming the role of residual plastic shear displacements along the crack wake in producing closure. It may be seen that the predominant closure process is in fact closely analogous to the plasticity induced closure in mode I loading, although it does rely on crack path roughness to generate the necessary shear displacements. It is important to note that plane stress conditions are not particularly necessary to the asperity contact process shown in Fig. 9 (i.e. as seen in mode I plasticity induced closure), as the critical deformations are shear in nature and do not require through-thickness contraction for volume conservation.

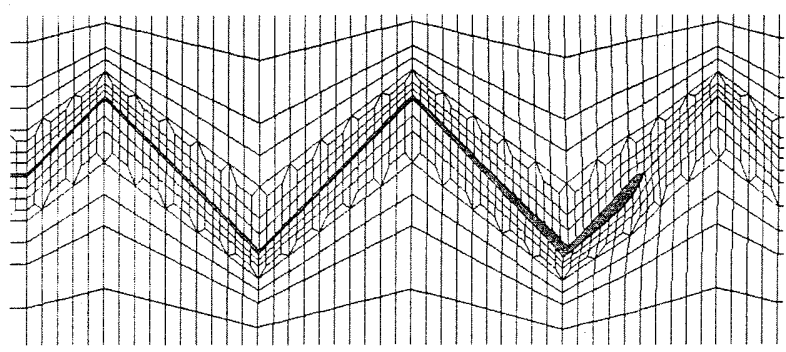

Fig. 10. Residual deformation of a deflected crack loaded and unloaded once. 


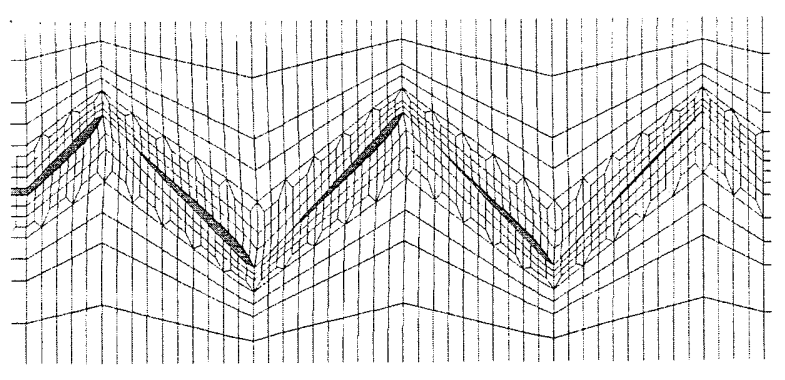

Fig. 11. Residual deformation of a crack loaded and unloaded at the turning points. Final loading and unloading performed elastically.

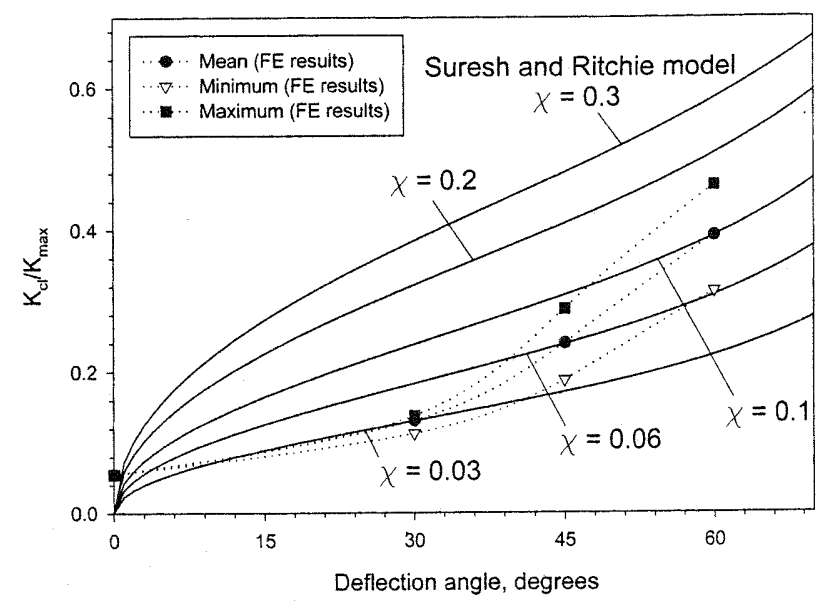

Fig. 12. Comparison of the present finite element results to the analytical model of Suresh and Ritchie [35].

Table 1

Values of $\chi$ evaluated from the finite element results

\begin{tabular}{lll}
\hline $\begin{array}{l}\text { Deffection angle } \\
\left({ }^{\circ}\right)\end{array}$ & $\chi$ At first asperity & $\begin{array}{l}\chi \text { Required to fit Suresh } \\
\text { model }\end{array}$ \\
\hline 30 & 0.047 & $0.0225-0.0335$ \\
45 & 0.055 & $0.0365-0.0885$ \\
60 & 0.27 & $0.0675-0.170$ \\
\hline
\end{tabular}

The dependence on deflection angle of the closure levels may be compared to the simple analytical model of RICC of Suresh and Ritchie [35] for various values of $\chi$, as shown in Fig. 12 with the finite element results being represented by the minimum, maximum, and mean quasi-stabilised closure levels. It can be seen that there is no single value of $\chi$ which accurately reflects the dependence on the deflection angle of the present results. However, given that the proposed mechanism by which closure is occurring is governed by local shear deformation effects, the value of $\chi$ would itself be expected to be dependent on the deflection angle; i.e. the extent of the net asperity offset in Fig. 9 will be dependent on the local mode II stress intensity factor range, which, under far-field mode I loading, will increase with increasing deflection angle. Values of $\chi$ have been evaluated from the finite element results, and are shown in Table 1. The data presented are for the first asperity behind the tip, at which closure occurs first and which can be expected to be the most important asperity in controlling the closure process. It can be seen that the actual values of $\chi$ are close to those required to produce comparable closure levels from the Suresh and Ritchie model, and approach the $\chi$ value of 0.3 suggested by Suresh and Ritchie for crack growth in high strength $\mathrm{Al}$ alloy plate.

The closure levels and the manner in which closure occurs in this model are somewhat different from the results of a similar study of RICC by Llorca [38]. However, it should be noted that the present work does not require the explanation of crack closure put forward by Llorca, whereby a change in the mixity from the loading cycle to the unloading cycle arises due to the intervening propagation of the crack, leading to a residual displacement of the crack flanks. This explanation is questionable to some extent, given that in a real near-threshold fatigue crack the increment of crack growth is very small in relation to typical crack deflection distances and, hence, the change in mixity during any load/unload cycle may be expected to be negligible.

In terms of the physical relevance of the present models it is of course difficult to compare the results to experimental data in the literature given the considerable variability that exists in closure measurement $[43,44]$, and the relatively idealised crack morphology used here. $\mathrm{Al}-\mathrm{Li}$ alloys are of some interest in this respect given the strong crystallographic texture levels commonly seen in wrought material and the propensity for crystallographic crack growth which may lead to unusually regular crack development, e.g. see Fig. 13, corresponding to crack growth in commercial AA8090 plate. Work by Liu et al. on TL orientation tests in brass textured plate has shown deflection angles to be very close to $60^{\circ}$ (due to the preferred $\{111\}$ plane orientations), with corresponding closure levels being measured as approximately 0.7 , consistent with similar data in the literature [3]. As such, the present $K_{\mathrm{cl}}$ predictions may be seen to be relatively low, although it is important to recognise that: (1) the present models do not include environmental contributions to crack tip irreversibility, (2) there is still a significant degree of crack path irregularity in Fig. 13, and (3) the results in Fig. 8 assume a simple homogeneous plastic deformation mode which clearly differs from the intrinsically heterogeneous slip band behaviour giving rise to the observed crack paths. Whilst the first two factors may contribute to the under prediction of closure levels, their effect cannot be explicitly quantified here.

In terms of deformation effects however, the influence of a simulated slip band ahead of the crack tip is shown in Fig. 14, for a $60^{\circ}$ deflected crack after the onset of closure, with the constant $\lambda$ set to $1 / 2$, i.e. $\tau_{0}$ in 


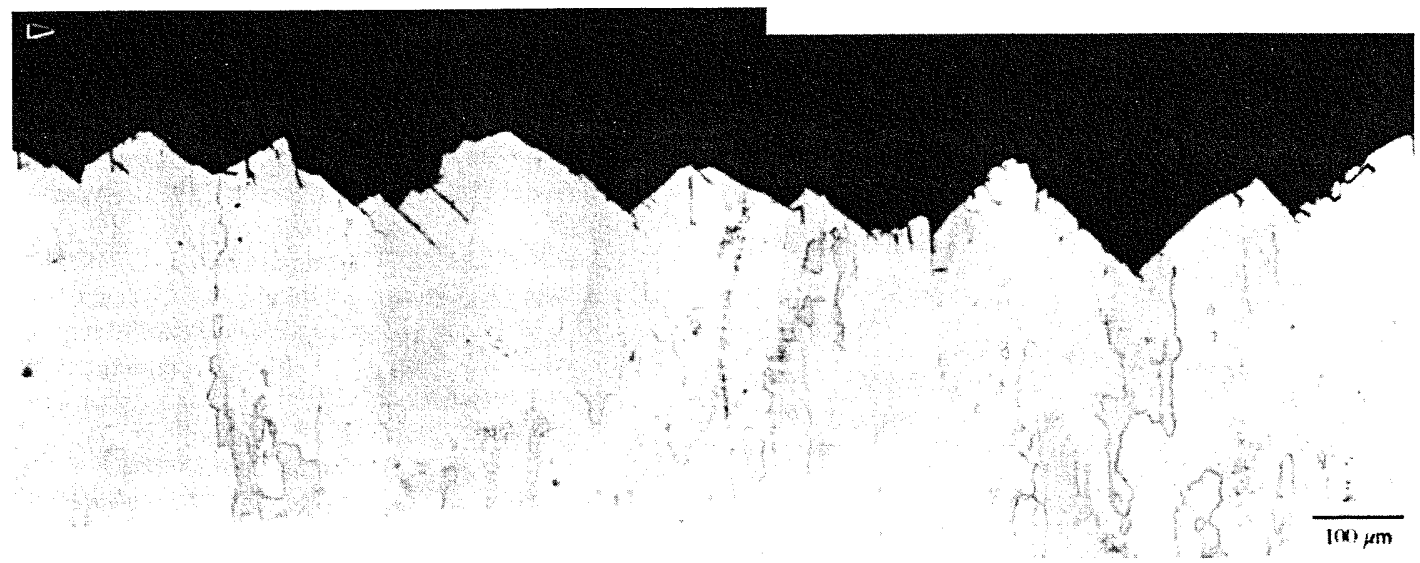

Fig. 13. Regular fatigue crack path deflection arising from strong crystallographic texture levels in $8090 \mathrm{Al}-\mathrm{Li}$ alloy tested in the LT orientation (arrow indicates crack growth direction).

the slip band is half that of the bulk material. It can be seen that ahead of the crack tip a significant residual shear offset has developed (cf. Fig. 1). The effect that this offset has on the closure levels can be seen by comparison of the predictions of the slip band model to those of the standard models, as shown in Fig. 15. For the $30^{\circ}$ deflected crack the effect of the simulated slip band is fairly small, leading to an $18 \%$ increase in the maximum closure stress. With increasing angle the effect becomes more significant leading to a $40 \%$ increase in closure levels for the $60^{\circ}$ deflected crack. In all cases the closure levels return to those of the standard model immediately prior to a deflection. This is due to the active slip band effectively ending at the next deflection point, therefore, as the crack approaches the next deflection point the slip band effect is essentially removed by the surrounding constraint. Whilst this represents a quite simple approximation to the presence of a strain softened slip band ahead of a crack tip, it may be seen that there is an increase in closure levels to values which are more consistent with those determined experimentally.

An investigation was also carried out into the dependence of the closure behaviour on the relative strength of the slip band. The analysis was performed for the $45^{\circ}$ deflected crack with values of $\lambda$ ranging from 1 (i.e. the band is effectively as strong as the bulk material) to 0.01 (i.e. the yield stress in the band is just $1 \%$ of that of the bulk material). The results are shown in Fig. 16 in terms of the mean closure stress intensity factor. It is clear that as the relative strength of the band diminishes there is an increase in the observed closure levels resulting from greater shear offset at the tip. It is important to note that the strain fields associated with a slip band will not be a simple function of the relative yield stress of the slip band and bulk material, but rather are a complex interaction between precipitate shear and substructure devel- opment. The present $\lambda$ parameter should be seen as an indicator of strain localisation and not as a full physical description of the process.

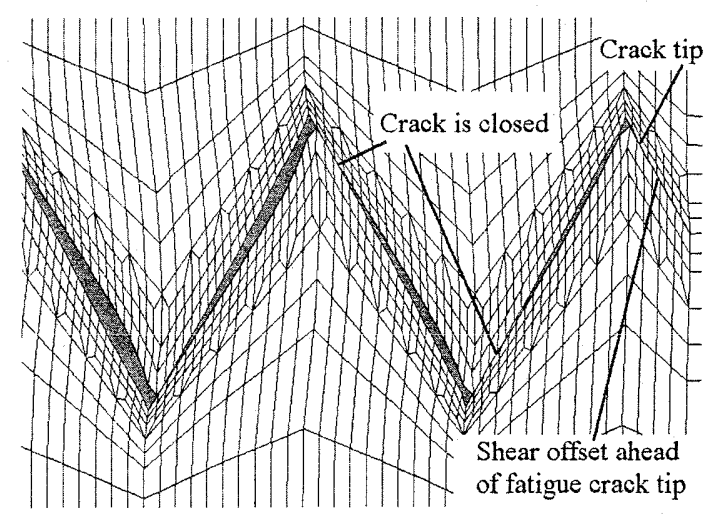

Fig. 14. Plot of the deformed mesh for a $60^{\circ}$ deflected crack with a slip band ahead of the crack tip enhancing the shear offset of the fracture surfaces.

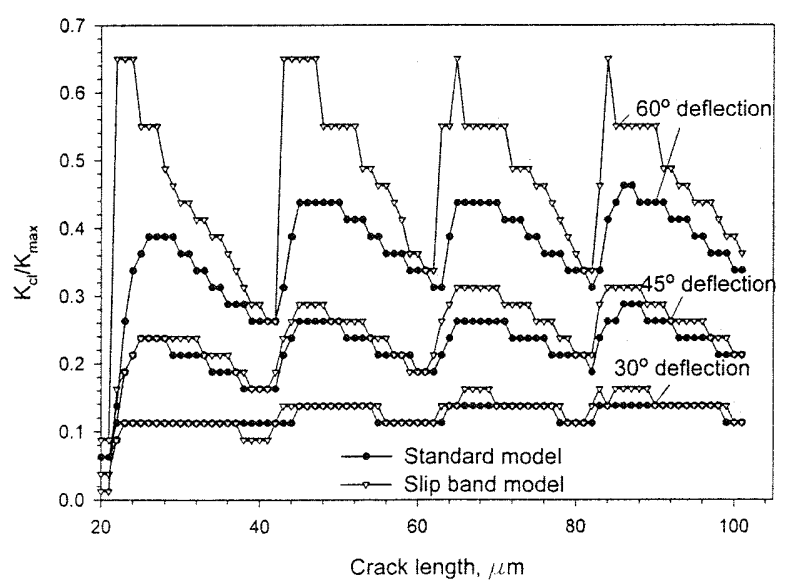

Fig. 15. Effect of slip localisation on the closure level for deflected crack geometries. 


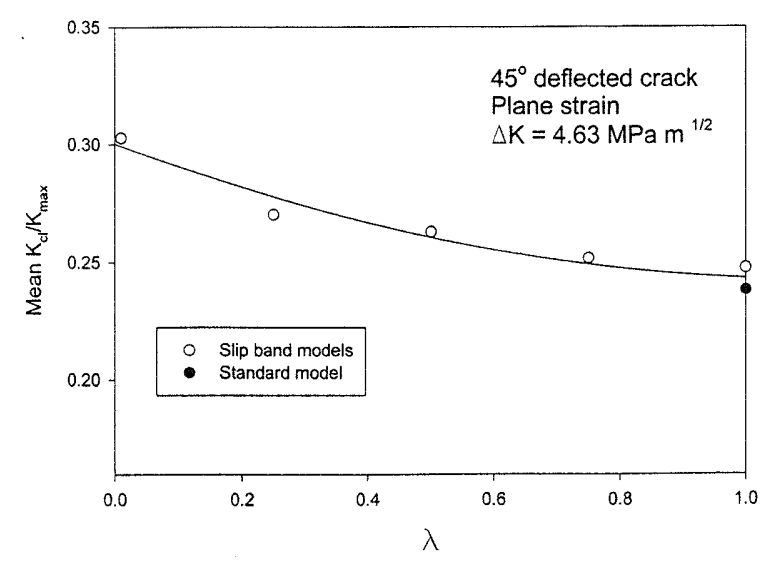

Fig. 16. Effect of the ratio of slip band yield stress to bulk material yield stress in the mean closure level for a $45^{\circ}$ deflected crack.

\section{Conclusions}

(1) Existing finite element techniques have been extended to investigate crack closure arising from crack deflection and plasticity. (2). Detailed examination of the closure process in deflected cracks has lead to the adoption of a closure criterion based on contact away from the immediate crack tip area associated with the onset of non-linearity in the specimen compliance, rather than the potentially anomalous behaviour of the first node behind the crack tip. (3). Periodic crack deflection has been shown to significantly increase crack closure levels in plane strain, with the effect increasing with deflection angle in general agreement with Suresh and Ritchie's analytical model. (4). The closure mechanism has been shown to be due to the local residual strains arising from the crack propagation process, rather than 'global' shear displacements due to mixed-mode behaviour at the crack tip. (5). A novel technique for simulating the effect of a planar slip band ahead of a fatigue crack has been constructed. The presence of these bands has been shown to significantly enhance predicted closure levels, consistent with those determined experimentally.

\section{References}

[1] V.K. Saxena, V.M. Radhakrishnan, Mater. Sci. Technol. 14 (1998) $1227-1241$.

[2] J.C. Newman, Jr., in: J.H. Benyon, M.W. Brown, T.C. Lindley, R.A. Smith, B. Tomkins (Eds.), Engineering Against Fatigue, Sheffield, UK, 17-21 March 1997, A.A. Balkema, Rotterdam, 1999, p. 261.

[3] K.T. Venkateswara Rao, R.O. Ritchie, Int. Mater. Rev. 37 (1992) $153-185$.

[4] A.K. Vasudevan, K. Sadananda, N. Louat, Scripta Metall. Mater. 27 (1992) 1673-1678

[5] N. Louat, K. Sadananda, M. Duesbury, A.K. Vasudevan, Metall. Trans. 24A (1993) 2225-2232.

[6] W. Elber, Eng. Fract. Mech. 2 (1970) 37-45.

[7] R.O. Ritchie, Mater. Sci. Eng. A103 (1988) 15-28.

[8] N. Walker, C.J. Beevers, Fatigue Eng. Mater. Struct. 1 (1979) $135-148$.
[9] Y. Brechet, F. Louchet, C. Marchionni, J.L. Verger-Gaugry, Phil. Mag. A56 (1987) 353-366.

[10] K. Minakawa, A.J. McEvily, Scripta Metall. 15 (1981) 633-636.

[11] A.F. Blom, A. Hadrboletz, B. Weiss, in: J. Carlsson, N.G. Ohlson (Eds.), Fourth International Conference on Mechanical Behaviour of Materials. Stockholm, Sweden, 1983, Pergamon, Oxford, 1983, p. 755

[12] J.C. Newman Jr, H. Armen, AIAA J. 13 (1975) 1017-1023.

[13] K. Ohji, K. Ogura, Y. Ohkubo, Int. J. Fract. 10 (1974) 123-124.

[14] R.C. McClung, H. Sehitoglu, Eng. Fract. Mech. 33(1989) 237-252.

[15] K. Ogura, K. Ohji, in: D.M.R. Taplin (Ed.), Proceedings of the Fourth International Conference on Fracture, University of Waterloo, Canada, June 1977, Pergamon, New York, 1978, p. 1035.

[16] A.F. Blom, D.K. Holm, Eng. Fract. Mech. 22 (1985) 997-1011.

[17] N.A. Fleck, J.C. Newman, Jr., in: J.C. Newman Jr., W. Elber(Eds.), Mechanics of Fatigue Crack Closure, ASTM STP 982, American Society for Testing and Materials, Philadelphia, PA, 1988, p. 319.

[18] R.C. McClung, B.H. Thacker, S. Roy, Int. J. Fract. 50 (1991) $27-49$.

[19] H. Sehitoglu, W. Sun, ASME J, Eng. Mater. Technol. 113 (1991) $31-40$.

[20] J. Llorca, V. Sanchez Galvez, Eng. Fract. Mech. 37 (1990) 185-196.

[21] L.W. Wei, M.N. James, Engng. Fract. Mech. 66 (1999) 223-242.

[22] D.F. Socie, Eng. Fract. Mech. 9 (1977) 849-865.

[23] H. Nakamura, H. Kobayashi, S. Yanase, H. Nakazawa, in: J. Carlsson, N.G. Ohlson (Eds.), Fourth International Conference on Mechanical Behaviour of Materials. Stockholm, Sweden, 1983, Oxford: Pergamon, Oxford, 1983, p. 817.

[24] N.E. Ashbaugh, B. Dattaguru, M. Khobaib, et al., Fatigue Fract. Eng. Mater. Struct. 20 (1997) 963-974.

[25] R.O. Ritchie, W. Yu, A.F. Blom, D.K. Holm, Fatigue Fract. Eng. Mater. Struct. 10 (1987) 343-362.

[26] P.L. Lalor, H. Sehitoglu, in: J.C. Newman Jr., W. Elber (Eds.), Mechanics of Fatigue Crack Closure, ASTM STP 982, American Society for Testing and Materials, Philadelphia, PA, 1988, p. 342.

[27] N.A. Fleck, Eng. Fract. Mech. 25 (1986) 441-449.

[28] S.B. Biner, O. Buck, W.A. Spitzig, Eng. Fract. Mech. 47 (1994) $1-12$.

[29] R. Pippan, F.O. Riemelmoser, Eng. Fract. Mech. 60 (1998) 315322.

[30] F.O. Riemelmoser, R. Pippan, Fatigue Fract. Eng. Mater. Struct. 21 (1998) 1425-1433.

[31] R.O. Ritchie, W. Yu, A.F. Blom, D.K. Holm, Fatigue Fract. Eng. Mater. Struct. 12 (1989) 73-75.

[32] K. Minakawa, G. Levan, A.J. McEvily, Metall. Trans. 17A (1986) 1787-1795.

[33] N.A. Fleck, R.A. Smith, Int. J. Fatigue 4 (1982) 157-160.

[34] G.H. Bray, A.P. Reynolds, E.A. Starke Jr, Metall. Trans. 23A (1992) 3055-3066.

[35] S. Suresh, R.O. Ritchie, Metall. Trans. 13A (1982) 1627-1631.

[36] J. Wasen, K. Hamberg, B. Karlsson, Mater. Sci. Eng. A102 (1988) $217-226$.

[37] S.H. Wang, C. Muller, H.E. Exner, Metall. Mater. Trans. 29A (1998) 1933-1939.

[38] J. Llorca, Fatigue Fract. Eng. Mater. Struct. 15 (1992) 655-669.

[39] ABAQUS v5.8, Hibbitt, Karlsson \& Sorensen, Inc., Rhode Island, USA, 1998.

[40] E 647 Volume 03.01, 1996 Annual Book of ASTM Standards, American Society for Testing and Materials, Philadelphia, PA, 1996, p. 570

[41] J.C. Nagtegaal, D.M. Parks, J.R. Rice, Comput. Methods Appl. Mech. Eng. 4 (1974) 153-177.

[42] C.M Ward-Close, R.O. Ritchie, in: J.C. Newman Jr., W. Elber (Eds.), Mechanics of Fatigue Crack Closure, ASTM STP 982, American Society for Testing and Materials, Philadelphia, PA, 1988, p. 93.

[43] E.P. Phillips, NASA TM 101601, 1989.

[44] E.P. Phillips, NASA TM 109032, 1993. 